\title{
Optimal Strong Parity Edge-Coloring of Complete Graphs
}

\author{
David P. Bunde*, Kevin Milans ${ }^{\dagger}$, Douglas B. West ${ }^{\ddagger}$, Hehui Wu ${ }^{\S}$
}

\begin{abstract}
A parity walk in an edge-coloring of a graph is a walk along which each color is used an even number of times. Let $p(G)$ be the least number of colors in an edge-coloring of $G$ having no parity path (a parity edge-coloring). Let $\widehat{p}(G)$ be the least number of colors in an edge-coloring of $G$ having no open parity walk (a strong parity edge-coloring). Always $\widehat{p}(G) \geq p(G) \geq \chi^{\prime}(G)$. We prove that $\widehat{p}\left(K_{n}\right)=2^{\lceil\lg n\rceil}-1$ for all $n$. The optimal strong parity edge-coloring of $K_{n}$ is unique when $n$ is a power of 2 , and the optimal colorings are completely described for all $n$.
\end{abstract}

\section{Introduction}

Our work began by studying which graphs embed in the hypercube $Q_{k}$, the graph with vertex set $\{0,1\}^{k}$ in which vertices are adjacent when they differ in exactly one coordinate. Coloring each edge with the position of the bit in which its endpoints differ yields two necessary conditions for the coloring inherited by a subgraph $G$ :

1) every cycle uses each color an even number of times,

2) every path uses some color an odd number of times.

Existence of a $k$-edge-coloring satisfying conditions (1) and (2) is also sufficient for a connected graph $G$ to be a subgraph of $Q_{k}$. This characterization of subgraphs of $Q_{k}$ appeared in 1972 (Havel and Movárek [7]). The problem was studied as early as 1953 (Shapiro [11]).

Let the usage of a color on a walk be the parity of the number of times it appears as the walk is traversed. A parity walk is a walk in which the usage of every color is even.

${ }^{*}$ Computer Science Department, Knox College, Galesburg, IL, dbunde@knox.edu. Partially supported by NSF grant CCR 0093348.

${ }^{\dagger}$ Department of Computer Science, University of Illinois, Urbana IL, milans@cs.uiuc.edu

$\ddagger$ Department of Mathematics, University of Illinois, Urbana, IL, west@math.uiuc.edu. Work supported in part by the NSA under Award No. MDA904-03-1-0037.

${ }^{\S}$ Department of Mathematics, University of Illinois, Urbana, IL. 
Condition (1) above states that every cycle is a parity walk, and (2) states that no path is a parity walk.

In general, a parity edge-coloring is an edge-coloring with no parity path, and a strong parity edge-coloring (spec) is an edge-coloring with no open parity walk (that is, every parity walk is closed). Some graphs embed in no hypercube, but giving the edges distinct colors produces a spec for any graph. Hence the parity edge-chromatic number $p(G)$ and the strong parity edge-chromatic number $\widehat{p}(G)$, defined respectively to be the minimum numbers of colors in a parity edge-coloring of $G$ and in a spec of $G$, are well defined. Elementary results on these parameters appear in [4].

When $T$ is a tree, $\widehat{p}(T)=p(T)=k$, where $k$ is the least integer such that $T$ embeds in $Q_{k}$. Since incident edges of the same color would form a parity path of length 2, every parity edge-coloring is a proper edge-coloring, and hence $p(G) \geq \chi^{\prime}(G)$, where $\chi^{\prime}(G)$ denotes the edge-chromatic number. Although there are graphs $G$ with $\widehat{p}(G)>p(G)$ [4], it remains unknown how large $\widehat{p}(G)$ can be when $p(G)=k$. It also remains unknown whether there is a bipartite graph $G$ with $\widehat{p}(G)>p(G)$.

When $n$ is a power of 2, we will prove that the complete graph $K_{n}$ has a unique optimal spec (up to isomorphism), which will help us determine $\widehat{p}\left(K_{n}\right)$ for all $n$. With a suitable naming of the vertices, we call this edge-coloring of $K_{n}$ the "canonical" coloring.

Definition 1.1 For $A \subseteq \mathbb{F}_{2}^{k}$, let $K(A)$ be the complete graph with vertex set $A$. The canonical coloring of $K(A)$ is the edge-coloring $f$ defined by $f(u v)=u+v$, where $u+v$ denotes binary vector addition. When $n=2^{k}$, letting $A=\mathbb{F}_{2}^{k}$ yields the canonical coloring of $K_{n}$.

Lemma 1.2 For $A \subseteq \mathbb{F}_{2}^{k}$, the canonical coloring of $K(A)$ is a spec. Consequently, if $n=2^{k}$, then $\widehat{p}\left(K_{n}\right)=p\left(K_{n}\right)=\chi^{\prime}\left(K_{n}\right)=n-1$.

Proof. If $W$ is an open walk, then its endpoints differ in some bit $i$. Thus in the canonical coloring the total usage of colors flipping bit $i$ along $W$ is odd, and hence some color has odd usage on $W$. The canonical coloring of $K\left(\mathbb{F}_{2}^{k}\right)$ uses $2^{k}-1$ colors (the color $0^{k}$ is not used). The lower bound follows from $\widehat{p}(G) \geq p(G) \geq \chi^{\prime}(G) \geq \Delta(G)$.

Since every complete graph is a subgraph of the next larger complete graph, we obtain $\widehat{p}\left(K_{n}\right) \leq 2^{\lceil\lg n\rceil}-1$. In Section 2 , we use linear algebra to prove our main result, showing that this upper bound is exact.

The canonical coloring is relevant to a less restrictive edge-coloring problem. A walk of length $2 k$ is repetitive if, for each $1 \leq i \leq k$, the $i$ th and $(k+i)$ th edges have the same color. A Thue coloring is an edge-coloring having no repetitive path, and the Thue number $t(G)$ is the minimum number of colors in a Thue coloring of $G$. Every parity edge-coloring is a Thue coloring, so always $t(G) \leq p(G)$. Alon, Grytczuk, Hałuszczak, and Riordan [2] observed that the canonical coloring yields $t\left(K_{n}\right) \leq 2^{\lceil\lg n\rceil}-1$. It seems that no good lower 
bounds on $t\left(K_{n}\right)$ are known. Our result implies that a Thue coloring of $K_{n}$ better than the canonical coloring must contain an open parity walk.

To further motivate our focus on complete graphs, we show that our main result strengthens a special case of Yuzvinsky's Theorem on sums of binary vectors. To state it, we need the Hopf-Stiefel function from the theory of quadratic forms.

Definition 1.3 (Hopf [8], Stiefel [12]) For positive integers $r$ and $s$, define $r \circ s$ to be the least integer $n$ such that $(x+y)^{n}$ is in the ideal of $\mathbb{F}_{2}[x, y]$ generated by $x^{r}$ and $y^{s}$.

In non-algebraic language, the definition has the following equivalent phrasing: $r \circ s$ is the least $n$ such that $\left(\begin{array}{l}n \\ k\end{array}\right)$ is even for each $k$ with $n-s<k<r$. The condition becomes vacuous if $n \geq r+s-1$, so trivially $r \circ s \leq r+s-1$.

Theorem 1.4 (Yuzvinsky [13]) For $A, B \subseteq \mathbb{F}_{2}^{k}$, let $C=\{a+b: a \in A, b \in B\}$. If $|A|=r$ and $|B|=s$, then $|C| \geq r \circ s$.

Generalizations and alternative proofs of Yuzvinsky's Theorem appear in [1], [3], [5]. The theorem is related to our results via a simple formula for the Hopf-Stiefel function recently proved by Plagne [10]. Subsequently, Károlyi [9] gave a short inductive proof. See [6] for a thorough survey of alternative formulas, related results, and generalizations.

Theorem 1.5 (Plagne [10], Károlyi [9]) $r \circ s=\min _{k \in \mathbb{N}}\left\{2^{k}\left(\left\lceil\frac{r}{2^{k}}\right\rceil+\left\lceil\frac{s}{2^{k}}\right\rceil-1\right)\right\}$.

When $A=B$ and both have size $r$, the minimization yields $r \circ r=2^{\lceil\lg r\rceil}$. Yuzvinsky's Theorem for this case says that every canonical coloring of $K_{r}$ uses at least $2^{\lceil\lg r\rceil}-1$ colors. Our result shows that in the more general family of strong parity edge-colorings, it remains true that at least $2^{\lceil\lg r\rceil}-1$ colors are needed.

The canonical coloring extends to complete bipartite graphs in a natural way: if $A, B \subseteq$ $\mathbb{F}_{2}^{k}$ and $K(A, B)$ is the complete bipartite graph with partite sets $A$ and $B$, then the edgecoloring defined by $f(a b)=a+b$ is a spec. The bound in Yuzvinsky's Theorem is always tight (see [5]); that is, for $r, s \leq 2^{k}$ there exist $A, B \subseteq \mathbb{F}_{2}^{k}$ with $|A|=r,|B|=s$, and $|C|=r \circ s$. Consequently, $\widehat{p}\left(K_{r, s}\right) \leq r \circ s$. We conjecture that equality holds. A direct proof in the graph-theoretic setting would strengthen all cases of Yuzvinsky's Theorem.

Conjecture $1.6 \widehat{p}\left(K_{r, s}\right)=r \circ s$.

\section{The Lower Bound}

In this section, we use linear algebra to prove that $\widehat{p}\left(K_{n}\right) \geq 2^{\lceil\lg n\rceil}-1$. The main idea is to introduce an additional vertex without needing additional colors until a power of 2 is reached. We begin by proving that every optimal spec of $K_{n}$ is a canonical coloring when $n$ is a power of 2 . 
Definition 2.1 An edge-coloring $f$ of a graph $G$ satisfies the 4-constraint if whenever $f(u v)=f(x y)$ and $v x \in E(G)$, also $u y \in E(G)$ and $f(u y)=f(v x)$.

Lemma 2.2 If $f$ is a parity edge-coloring in which every color class is a perfect matching, then $f$ satisfies the 4-constraint.

Proof. Otherwise, given $f(u v)=f(x y)$, the edge of color $f(v x)$ incident to $u$ forms a parity path of length 4 with $u v, v x$, and $x y$.

Theorem 2.3 If $f$ is a parity edge-coloring of $K_{n}$ in which every color class is a perfect matching, then $f$ is a canonical coloring and $n$ is a power of 2 .

Proof. Every edge is a canonically colored copy of $K_{2}$. Let $R$ be a largest vertex set such that $|R|$ is a power of 2 and $f$ restricts to a canonical coloring on $R$. Define $j$ by $|R|=2^{j-1}$. We are given a bijection $\phi$ from $R$ to $\mathbb{F}_{2}^{j-1}$ under which $f$ is the canonical coloring.

Since $f$ is canonical, every color used within $R$ by $f$ forms a matching of $R$. Let $c$ be a color not used within $R$; since $c$ is used on a perfect matching, $c$ matches $R$ to some set $U$. Let $R^{\prime}=R \cup U$. Define $\phi^{\prime}: R^{\prime} \rightarrow \mathbb{F}_{2}^{j}$ as follows: for $x \in R$, obtain $\phi^{\prime}(x)$ by appending 0 to $\phi(x)$; for $x \in U$ obtain $\phi^{\prime}(x)$ by appending 1 to $\phi\left(x^{\prime}\right)$, where $x^{\prime}$ is the neighbor of $x$ in color $c$. Within $R^{\prime}$, we henceforth refer to the vertices by their names under $\phi^{\prime}$.

By Lemma 2.2, the 4-constraint holds for $f$. The 4-constraint copies the coloring from the edges within $R$ to the edges within $U$. To see this, consider $x^{\prime}, y^{\prime} \in U$ arising from $x, y \in R$, with $f\left(x x^{\prime}\right)=f\left(y y^{\prime}\right)=c$. Now $f\left(x^{\prime} y^{\prime}\right)=f(x y)=x+y=x^{\prime}+y^{\prime}$, using the 4-constraint, the fact that $f$ is canonical on $R$, and the definition of $\phi^{\prime}$. Hence $f$ is canonical within $U$.

Finally, let $u$ be the name of the color on the edge $0^{j} u$, for $u \in U$. For any $v \in R$, let $w=u+v$; note that $w \in U$. Both $0^{j} v$ and $u w$ have color $v$, since $f$ is canonical within $R$ and within $U$. By applying the 4-constraint to $\left\{v 0^{j}, 0^{j} w, w u\right\}$, we conclude that $f(u v)=f\left(0^{j} w\right)=w$. Since $w=u+v$, this completes the proof that $f$ is canonical on $R^{\prime}$.

In connection with this uniqueness result, Mubayi asked whether a stability property holds. That is, when $n$ is a power of 2 , does there exist a parity edge-coloring or a spec of $K_{n}$ that has only $(1+o(1)) n$ colors but is "far" from the canonical coloring?

The main result needs several algebraic observations. Relative to any $k$-edge-coloring $f$, the parity vector $\pi(W)$ of a walk $W$ is the binary $k$-tuple whose $i$ th bit agrees in parity with the usage of color $i$ along $W$. Let the parity space $L_{f}$ be the set of parity vectors of closed walks. We note that $L_{f}$ is a vector space.

Lemma 2.4 If $f$ is an edge-coloring of a connected graph $G$, then $L_{f}$ is a binary vector space. 
Proof. Since $L_{f} \subseteq \mathbb{F}_{2}^{k}$, it suffices to show that $L$ is closed under addition. Given a $u, u$ walk $W$ and a $v, v$-walk $W^{\prime}$, let $P$ be a $u, v$-path in $G$, and let $\bar{P}$ be its reverse. Following $W, P, W^{\prime}, \bar{P}$ in succession yields a $u, u$-walk with parity vector $\pi(W)+\pi\left(W^{\prime}\right)$.

For a vector space $L$, let $w(L)$ denote the least number of nonzero coordinates of any vector in $L$ (set $w(L)=\infty$ if $L=\{0\})$. For an edge-coloring $f$ of $K_{n}, w\left(L_{f}\right)$ determines whether $f$ is a spec.

Lemma 2.5 If an edge-coloring $f$ of a graph $G$ is a spec, then $w\left(L_{f}\right) \geq 2$. The converse holds when $G=K_{n}$.

Proof. If the parity vector of a closed walk $W$ has weight 1 , then one color has odd usage in $W$ (say on edge $e$ ). Now $W-e$ is an open parity walk, and $f$ is not a spec.

If $f$ is not a spec, then $\pi\left(W^{\prime}\right)=0$ for some open walk $W^{\prime}$. In $K_{n}$, the ends of $W^{\prime}$ are adjacent, and adding that edge yields a closed walk whose parity vector has weight 1 .

Lemma 2.6 For any colors $a$ and $b$ in an optimal spec $f$ of $K_{n}$, there is some closed walk $W$ on which the colors having odd usage are $a, b$, and one other.

Proof. We use Lemma 2.5 repeatedly. Since $f$ is optimal, merging the colors $a$ and $b$ into a single color $a^{\prime}$ yields an edge-coloring $f^{\prime}$ that is not a spec. Hence under $f^{\prime}$ there is a closed walk $W$ on which $f^{\prime}$ has odd usage for only one color $c$. Also $c \neq a^{\prime}$, since otherwise $f$ has odd usage on $W$ for only $a$ or $b$. With $c \neq a^{\prime}$ and the fact that $f$ has odd usage for at least two colors on $W$, both $a$ and $b$ also have odd usage on $W$, and $W$ is the desired walk.

The same idea as in Lemma 2.6 shows that $w\left(L_{f}\right) \geq 3$ when $f$ is an optimal spec of $K_{n}$, but we do not need this observation. We note, however, that the condition $w\left(L_{f}\right) \geq 3$ is the condition for $L_{f}$ to be the set of codewords for a 1-error-correcting code. Indeed, when $n=2^{k}$ and $f$ is the canonical coloring, $L_{f}$ is a perfect 1-error-correcting code of length $n-1$.

A dominating vertex in a graph is a vertex adjacent to all others. We use $d_{H}(v)$ and $N_{H}(v)$ to denote the degree and neighborhood of a vertex $v$ in a graph $H$.

Lemma 2.7 If $f$ is an edge-coloring of a graph $G$ with a dominating vertex $v$, then $L_{f}$ is the span of the parity vectors of triangles containing $v$.

Proof. By definition, the span is contained in $L_{f}$. Conversely, consider any $\pi(W) \in L_{f}$. Let $S$ be the set of edges with odd usage in $W$, and let $H$ be the spanning subgraph of $G$ with edge set $S$. Since the total usage at each vertex of $W$ is even, $H$ is an even subgraph of $G$. Hence $H$ decomposes into cycles, which are closed walks, and $\pi(W)$ is the sum of the parity vectors of these cycles.

It therefore suffices to show that $S$ is the set of edges that appear in an odd number of the triangles formed by $v$ with edges of $H-v$. Each edge of $H-v$ is in one such triangle, so 
we need consider only edges involving $v$. An edge $v w$ lies in an odd number of these triangles if and only if $d_{H-v}(w)$ is odd, which occurs if and only if $w \in N_{H}(v)$, since $d_{H}(w)$ is even. By definition, $v w \in E(H)$ if and only if $v w$ has odd usage in $W$ and hence lies in $S$.

Lemma 2.8 If $f$ is an optimal spec of $K_{n}$ that uses some color fewer than $n / 2$ times, then $f$ extends to a spec of $K_{n+1}$ using the same colors.

Proof. View $K_{n+1}$ as arising from $K_{n}$ by adding a vertex $u$. Let $a$ be a color used fewer than $n / 2$ times by $f$, and let $v$ be a vertex of $K_{n}$ at which $a$ does not appear.

We use $f$ to define $f^{\prime}$ on $E\left(K_{n+1}\right)$. Let $f^{\prime}$ agree with $f$ on $E\left(K_{n}\right)$, and let $f^{\prime}(u v)=a$. To define $f^{\prime}$ on each remaining edge $u w$, first let $b=f(v w)$. By Lemma 2.6, there is a closed walk $W$ with odd usage precisely for $a$ and $b$ and some third color $c$ under $f$. Let $f^{\prime}(u w)=c$.

Note that $f^{\prime}$ uses the same colors as $f$. It remains only to show that $f^{\prime}$ is a spec. To do this we prove that $w\left(L_{f^{\prime}}\right) \geq 2$, by showing that $L_{f^{\prime}} \subseteq L_{f}$. By Lemma 2.7, it suffices to show that $\pi(T) \in L_{f}$ when $T$ is a triangle in $K_{n+1}$ containing $v$.

Triangles not containing $u$ lie in the original graph and have parity vectors in $L_{f}$. Hence we consider the triangle $T$ formed by $\{u, v, w\}$. Now $\pi(T)=\pi(W) \in L_{f}$, where $W$ is the walk used to specify $f^{\prime}(u w)$.

Theorem $2.9 \widehat{p}\left(K_{n}\right)=2^{\lceil\lg n\rceil}-1$.

Proof. If some color class in an optimal spec is not a perfect matching, then $\widehat{p}\left(K_{n}\right)=$ $\widehat{p}\left(K_{n+1}\right)$, by Lemma 2.8. This vertex absorption cannot stop before the number of vertices reaches a power of 2 , because when every color class is a perfect matching the coloring is canonical, by Theorem 2.3. It cannot continue past $2^{\lceil\lg n\rceil}$ vertices, since the maximum degree is a lower bound on $\chi^{\prime}$ and $\widehat{p}$. Hence $\widehat{p}\left(K_{n}\right)=\widehat{p}\left(K_{2^{[\lg n\rceil}}\right)=2^{\lceil\lg n\rceil}-1$.

Corollary 2.10 If $f$ is an optimal spec of $K_{n}$, then $f$ is obtained by deleting vertices from the canonical coloring of $K_{2[\lg n]}$.

Proof. By Lemma 2.8, we may extend $f$ to an optimal spec $f^{\prime}$ of $K_{2[\lg n]}$; by Theorem 2.3, $f^{\prime}$ is the canonical coloring.

It is natural to wonder whether every edge-coloring of $K_{n}$ that satisfies the 4-constraint is a spec or a parity edge-coloring. One can construct examples that show the answer is no. Similarly, not every parity edge-coloring of $K_{n}$ is a spec. Nevertheless, it may be that every optimal parity edge-coloring is a spec. We offer the following conjecture, which in [4] we proved for $n \leq 16$.

Conjecture $2.11 p\left(K_{n}\right)=\widehat{p}\left(K_{n}\right)$ for every positive integer $n$. 


\section{Acknowledgement}

We thank Dan Cranston, Will Kinnersley, Brighten Godfrey, Michael Barrus, and Mohit Kumbhat for helpful discussions.

\section{References}

[1] N. Alon, Combinatorial Nullstellensatz. Combin., Prob., and Computing 8 (1999), 7-29.

[2] N. Alon, J. Grytczuk, M. Hałuszczak, and O. Riordan, Nonrepetitive colorings of graphs. Random Structures Algorithms 21 (2002), 336-346.

[3] B. Bollobás, and I. Leader, Sums in the grid. Discrete Math. 162 (1996), 31-48.

[4] D. P. Bunde, K. Milans, D. B. West, and H. Wu, Parity edge-coloring of graphs. preprint.

[5] S. Eliahou and M. Kervaire, Sumsets in vector spaces over finite fields. J. Number Theory 71 (1998), 12-39.

[6] S. Eliahou and M. Kervaire, Old and new formulas for the Hopf-Stiefel and related functions. Expo. Math. 23 (2005), 127-145.

[7] I. Havel and J. Movárek, B-valuation of graphs. Czech. Math. J. 22, 338-351.

[8] H. Hopf, Ein topologischer Beitrag zur reelen Algebra, Comment. Math. Helv. 13 (1940/41), 219-239.

[9] G. Károlyi, A note on the Hopf-Stiefel function. Europ. J. Combin. 27 (2006), 1135-1137.

[10] A. Plagne, Additive number theory sheds extra light on the Hopf-Stiefel o function. L'Enseignement Math. 49 (2003) 109-116.

[11] H. Shapiro, The embedding of graphs in cubes and the design of sequential relay circuits, Bell Telephone Laboratories Memorandum, July 1953.

[12] E. Stiefel, Über Richtungsfelder in den projektiven Räumen und einen Satz aus der reelen Algebra, Comment. Math. Helv. 13 (1940/41), 201-218.

[13] S. Yuzvinsky, Orthogonal pairings of Euclidean spaces. Michigan Math. J. 28 (1981), 109-119. 